\title{
Global change and the polar regions
}

\section{Chris Rapley, British Antarctic Survey}

The Earth System consists of the solid planet, its atmosphere, oceans, ice and biota. These are coupled via a myriad of complex physical, chemical and biological processes, which occur over a vast range of scales of time and space. Natural variability and evolution are intrinsic to the system's behaviour. However, recent dramatic surges in human population and economic activity have resulted in mankind becoming a new and potent agent of global-scale changes. For example, after 10000 years of relative stability, there is mounting evidence that human activities are causing global climatic change.

In order to balance the often conflicting requirements of economic growth and environmental protection, it is essential to understand the workings of the Earth System and the workings of human society within it. This can only be achieved through the methodology of science. Without science there can be no "sustainable development."

Although geographically remote, the polar regions feature centrally in many of the key contemporary Earth System issues. Thus:

- The seasonal growth and decay of sea ice and snow has a profound influence on the albedo and radiative balance of the planet. Snow/ ice-albedo feedback, in which the melting of sea ice or snow results in increased heat absorption. underlies the prediction by global climate models of enhanced polar warming. The strong positive temperature trend observed over the Antarctic Peninsula, and the associated much-publicized collapse of ice shelves, may be early manifestations of this.

- The polar ice sheets, lake sediments and marine sediments contain invaluable information on past states of the Earth System, providing crucial insights into its functioning, and the characteristics of "natural" change.

- Palaeo data of various forms indicate that the physical climate has the capacity for sudden and dramatic jumps. The only plausible mechanism for such events involves changes in the thermohaline circulation of the ocean, which themselves implicate the polar deep bottom water formation. Antarctic deep bottom water permeates beneath $40 \%$ of the world's oceans, but its formation mechanisms and role are poorly understood.

- The mass balance of the ice sheets is critical, since any variations will have a significant impact on mean sea level, and hence on coastlines world-wide.

- The dynamics and temperature structure of the polar stratosphere are such that the region is especially prone to the destruction of the ozone layer by man-made chemicals such as CFCs. Thus the "ozone hole" was first detected over Antarctica, and remains especially strong there, in spite of the international legal instruments introduced to ban CFC production and usage.

- Polar ecosystems tend to be simpler in structure than lower latitude systems, and their members exhibit many examples of specialized adaptations. Thus their study can provide fundamental insights.

- The Southern Ocean is one of the world's most productive oceans and is a potentially major fishery. The research undertaken to guide the Commission for the Conservation of Marine Living Resources (CCAMLR) has adopted an ecosystem approach and lies at the forefront of its field.

- Antarctica was the "hub" of the Gondwana supercontinent. Its study is essential if the mechanisms of continental break up and drift are to be fully understood.

- The convergence of magnetic field lines at the geographic poles provides the opportunity for the study of Sun-Earth interactions, which are important to understand the fundamentals of plasma magnetohydrodynamics, as well as being of practical value for radio communications and the design of robust satellite systems.

- The geographic remoteness of the polar regions results in very low levels of man-made pollu- 
tion. Nonetheless, pollutants can be detected there, providing a measure of the extent to which mankind is contaminating the planet.

New opportunities for studying the polar regions and cryospheric processes are provided by upcoming spacecraft from the USA, Europe and Japan, by the potential for greater coordination of existing national field stations and facilities, from advances in telecommunications technology, and from enhanced levels of international networking. Satellite remote sensing offers a special contribution, although to fulfil its potential, this needs to be coupled with dedicated field work.

The polar regions are hazardous to work in and costly to access. Hence, international collaborations are particularly valuable since they share costs, avoid unnecessary duplication and, through the establishment of a "critical mass", permit issues to be addressed which transcend the capability of any one nation. Examples include the projects organized by the International Arctic
Science Committee and the Scientific Committee on Antarctic Research in the area of global change. Other international organizations, such as the World Climate Research Programme and the International Geosphere-Biosphere Programme, also address polar issues.

The challenges facing the polar science community include:

- Carrying out a review or "reality check" on priorities;

- Rationalizing and strengthening research structures;

- Strengthening and sustaining research networks;

- Exploiting the new opportunities;

- Synthesizing "understanding" from the plethora of results.

This conference provides an excellent opportunity to hear the very latest results and to discuss and agree on specific priorities for the future. 